\title{
Significance of Regional Antibiogram and MDR of ESBL Producing Uropathogens Infecting Non-hospitalized Patients: Gurugram
}

\author{
Rishabh Rajput ${ }^{1,2 *}$ and Surendra Sarsaiya ${ }^{1,3}$ \\ ${ }^{1}$ Department of Microbiology, Sri Satya Sai University of Technology and Medical Sciences, \\ Sehore-Madhya Pradesh, India \\ ${ }^{2}$ Department of Microbiology, Modern Diagnostic and Research Centre, Gurugram, \\ Haryana, India \\ ${ }^{3}$ Key Laboratory of Basic Pharmacology of Ministry of Education, Zunyi Medical University, \\ Zunyi, Guizhou, China \\ *Corresponding author
}

A B S T R A C T

\section{Keywords}

UTI, Multi-drug resistant, Extendedspectrum $\beta$ lactamase, ESBL, E. coli, K. pneumoniae, CLSI, ATCC

Article Info

Accepted:

10 January 2018 Available Online:

10 February 2018
Worldwide, UTI is found to be the second most common form of infection ranging from neonate to geriatric age group, accounting for nearly $25 \%$ of all infections. It is the most common infection experienced by humans after respiratory and gastrointestinal infections Increase in extended-spectrum betalactamase (ESBL) producing organisms in recent years has led to the limitation of treatment option. This study was focused to investigate the prevalence of different uropathogens producing ESBL and its antibiogram in non-hospitalized patients. 1495 non-repetitive urine samples were collected in the study. A total of 335 (22.41\%) samples showed significant bacteriuria. Females were found to be more infected as compare to Males. The most affected age group was $\geq 60$ years. The most common ESBL producing uropathogens were E. coli followed by Klebsiella pneumoniae. All the ESBL positive isolates were found to be Multi-Drug resistant (MDR). The prevalence of MDR was also significantly increased due to ESBL production. International guidelines are no longer applicable for treating UTIs in India and development of specific guidelines based on local susceptibility patterns is an absolute necessity and researchers need to keep monitoring on the everchanging trend in the antibiotic susceptibility pattern of ESBL positive and negative isolates in our region and facilitate evidence-based judicious antibiotic use policy to treat UTI. Every healthcare setup should have their antibiogram policy to enable them to choose the right antibiotics as per the trend going in the particular area and should stop prescribing antibiotics without the culture and sensitivity test report. 


\section{Introduction}

Worldwide, Urinary tract infection (UTI) is found to be the second most common form of infection ranging from neonate to geriatric age group, ${ }^{1,2}$ accounting nearly $25 \%$ of all infections. Globally, approximately 150 million people are diagnosed with UTI each year, costing the global economy more than 6 billion US dollars ${ }^{3}$. The infection involves upper or lower urinary tract ${ }^{4}$. UTI is characterised by various conditions such as burning feeling during the passage of urine, lower abdominal pain, urgency in urination, fever, dysuria (Burning urination or painful urination), blood in the urine, feeling tired or shaky, pyuria ${ }^{5}$. It is the most common infection experienced by humans after respiratory and gastrointestinal infections. Gram-negative bacteria such as Escherichia coli (E. coli), Klebsiella pneumoniae (K. pneumoniae), Klebsiella oxytoca (K.oxytoca), Proteus mirabilis (P.mirabilis), P.aeruginosa (P.aeruginosa) and occasionally by other bacteria are the most common agents. The predominant uropathogens acquired from any source are Gram-negative bacteria with $E$. coli accounting for the highest prevalence in most instances ${ }^{6}$.

ESBL producing bacteria are those that hydrolyse the oxyimino beta-lactams and monobactams. However, they do not affect the cephamycins and carbapenems ${ }^{7}$. ESBLs are also able to hydrolyse 3 and 4 generation cephalosporins and monobactams. Lactamase inhibitors inhibit ESBL producing strains (clavulanic acid, sulbactam and tazobactam ${ }^{8-}$ ${ }^{10}$. It is increasing very rapidly and becoming the major problem in the area which is prone to infectious diseases.

The issues which are associated with the isolates producing ESBL are challenging to find or treated, thereby causing increased mortality of the patients ${ }^{11}$. During mid-1980's in Western Europe, after the initial discoveries of ESBL producing uropathogens, the occurrence increased steadily in each year. The prevalence of ESBL producing uroapthogens vary worldwide and is rapidly changing over time. ESBL was first identified among Klebsiella and subsequently, in E. coli, S.marcescens, P.aeruginosa and other gramnegative bacilli ${ }^{12,13}$. MDR has increased dramatically in recent years. There is growing concern of MDR gram-negative bacteria which produces ESBLs ${ }^{14}$.

Beta-lactam antibiotics such as oxyiminocephalosporins have been used for the treatment of Gram-negative bacterial infections since 1980's ${ }^{8}$. Unfortunately, nowadays, beta-lactamase resistance has been growing among members of Enterobacteriaceae, including E. coli and $K$. pneumoniae. The most common cause of beta-lactam resistance is beta-lactamase enzymes, which deactivate beta-lactam drugs by breaking down the beta-lactam ring ${ }^{15}$. ESBLs are a class of enzymes that are determined by the plasmid, which hydrolyses a wide variety of cephalosporins such as cefotaxime, ceftazidime, ceftriaxone and drugs that have the beta-lactam ring within their structure $^{16}$.

\section{Materials and Methods}

\section{Study design and area}

This prospective study was conducted from urine samples collected from non-hospitalized patients complaining about UTIs. The samples were processed in Bacteriology Section, Department of Microbiology, Modern Diagnostic and Research Centre, Gurugram, Haryana-India.

\section{Processing of the samples}

All samples were processed within 1-2 hours of the collection, and in case of delay, the specimens were refrigerated at $4^{\circ} \mathrm{C}$. All urine 
samples were cultured by the semiquantitative method. In short, $0.01 \mathrm{ml}$ of urine was inoculated on Cysteine lactose electrolyte deficient agar (CLED Agar) (Hi-media Pvt. Ltd) by crisscross streaking using disposable calibrated flexi-loop (Hi-media Pvt. Ltd) and incubated for $18-24$ hours at $37^{\circ} \mathrm{C}$ under aerobic conditions.

A pure growth of the gram-negative isolate on a colony count $\geq 10^{5}$ colony forming units was considered as significant bacteriuria. Isolation and identification of the strains were done following their morphology in Gram's staining, culture characteristics and biochemical properties. Plates with no growth were further incubated for another 24 hours before interpreting it as a negative culture.

\section{Quality control}

The bacterial suspension was prepared and was adjusted to a $0.5 \mathrm{McFarland}$ standard solution (Hi-media Pvt. Ltd). American Type Culture Collection (ATCC) standard reference strains $P$. aeruginosa ATCC-27853, S.aureus ATCC-25923, E. coli ATCC-25922 were used as a quality control strains for antimicrobial susceptibility testing. All the ATCC strains used in the current study were procured from Microbiologics, USA.

\section{Antibiotic sensitivity testing}

Antibiotic sensitivity testing was done by Kirby-Bauer disc diffusion method on Mueller-Hinton agar, and interpretation of the results was done as described by CLSI guidelines $^{17}$. Antibiotics discs (Hi-Media Lab Pvt. Ltd) used were Ampicillin (AMP)-10 $\mu \mathrm{g}$, Gentamicin (GEN)-10 $\mu \mathrm{g}$, Tobramycin (TOB)-10 $\mu \mathrm{g}, \quad$ Amikacin (AK)-10 $\mu \mathrm{g}$, Amoxicillin-Clavulanic Acid (AMC)-20/10 $\mu \mathrm{g}, \quad$ Ampicillin/Sulbactum (A/S)-20/10 $\mu \mathrm{g}$, Piperacillin/Tazobactum (P/T)-100/10 $\mu \mathrm{g}$, Cefuroxime (CXM)-30 $\mu \mathrm{g}$, Cefepime (CPM)$30 \mu \mathrm{g}$, Ceftazidime (CAZ)-30 $\mu \mathrm{g}$, Aztreonam
(AT)-15 $\mu \mathrm{g}, \quad$ Cefoxitin $\quad$ (CX)-30 $\mu \mathrm{g}$, Levofloxacin (LE)- $5 \mu \mathrm{g}$, Ciprofloxacin (CIP)$5 \mu \mathrm{g}$, Imipenem (IMP)-10 $\mu \mathrm{g}$, Ertapenem (ETP)-10 $\mu \mathrm{g}$, Cotrimoxazole (COT)-25 $\mu \mathrm{g}$, Tetracycline (TET)-30 $\mu \mathrm{g}$, Nitrofurantoin (NIT) $-300 \mu \mathrm{g}$.

\section{Screening test for ESBL production ${ }^{17}$}

The organism was swabbed onto the Mueller Hinton agar (MHA) plate. Antibiotic discs Ceftazidime with zone diameter of $\leq 22 \mathrm{~mm}$ and Cefotaxime with zone diameter of $\leq 27$ $\mathrm{mm}$ was considered indicative of suspicious for ESBL producer. These isolates were further subjected to the phenotypic confirmation testing ${ }^{17}$.

\section{Confirmatory test for ESBL production ${ }^{17}$}

ESBL productions among the potential ESBLproducing strains by screening method were confirmed by CLSI phenotypic confirmation method. Comparison of the zone of inhibition was made for the Ceftazidime $(30 \mu \mathrm{g})$ and Cefotaxime $(30 \mu \mathrm{g})$, discs alone with the Ceftazidime and Cefotaxime discs containing Clavulanic acid $(10 \mu \mathrm{g})$, ESBL production was confirmed by a $>5 \mathrm{~mm}$ increase in the zone diameter for either antibiotic tested in combination with Clavulanic acid against the zone diameter when tested alone confirmed the presence of ESBL production by that $\operatorname{organism}^{17}$.

The increase in zone diameter was due to the inhibition of the $\beta$-lactamase by Clavulanate. K. pneumoniae (ATCC-700603) was used as a positive control, and E. coli (ATCC-25922) was used as a negative control.

\section{Statistical analysis}

Chi-square test was used for statistical analysis of the data. A p-Value of less than 0.05 was considered as statistical significant. 


\section{Results and Discussion}

\section{Demographical features of the studied subjects}

In the current study, 1495 urine samples suspected of UTI were collected in sterile and leak-proof containers from non-hospitalized patients in and around Gurugram, Haryana. The median age was 40 years, and the age range was $0-89$ years. There were $77.59 \%$ (1160/1495) culture-negative samples, with the median age of 39 years and age range of 0 88 years. In the present study, $22.41 \%$ (335/1495) samples were found positive for urine culture with the median age of 47 years and age range of 0-89 years. Prevalence of UTI among the age wise distribution showed a significant difference $(\mathrm{p}=0.000)$. There were $42.61 \%(637 / 1495)$ males included in this study and $83.20 \%$ (530/637) individuals presented with the negative urine culture. Rest of the $16.80 \%$ $(107 / 637)$ males was presented with the positive urine culture. There were $57.39 \%$ (858/1495) females included in the study and $73.43 \%$ (630/858) individuals presented with the negative urine culture and rest of the $26.57 \%$ (228/858) females shown with the positive urine culture. Prevalence of UTI among the different gender showed a significant difference $(\mathrm{p}=0.000)($ Table 1$)$.

\section{ESBL status of the uropathogens}

In the present study, Detection of ESBL was done by CLSI screening test followed by confirmatory tests ${ }^{17}$. As per the recommendation of the CLSI guidelines, it was performed for Escherichia coli, $K$. pneumoniae, $K$. oxytoca and $P$. mirabilis. Among these, only E. coli and $K$. pneumoniae were found to be positive. A total number of 303 gram-negative uropathogens (Median age: 49; Range: 0-89) were isolated. Among them, $38.94 \%$ (118/303) were ESBL producers (Median age: 54; Range: $0-89$ ) and $47.85 \%$
(145/303) were non ESBL producers (Median age: 45 ; Range: 0-86 and others were $13.20 \%$ (40/303) (Median age: 47.5 ; Range:1-88). 234 (77.23\%) E. coli were isolated. The frequency of ESBL positive isolates were $47.44 \%$ (111/234), and ESBL negative isolates were 52.56\% (123/234). 27 (8.91\%) K. pneumoniae were isolated. The frequency of ESBL positive isolates were $25.93 \%$ (7/27), and ESBL negative isolates were $74.07 \%$ (20/27). $1(0.33 \%)$ Interestingly, all the P. mirabilis and $K$. oxytoca isolates were ESBL negative $(1,100 \%)$. In the current study, no significant statistical difference was found among ESBL negative and ESBL positive uropathogens ( $\mathrm{p}=$ 0.104). Other GNB's were not tested for ESBL production (Table 2).

There were $32.01 \%$ (97/303) males, in which $38.14 \%$ (37/97) isolates were ESBL positive, $42.27 \%$ (41/97) were ESBL negative and other GNB for which ESBL production was not tested were $19.59 \%$ (19/97). In females $67.99 \%$ (206/303), 39.32\% (81/206) isolates were ESBL positive, $50.49 \%$ (104/206) were ESBL negative, and other GNB for which ESBL production was not tested were $10.19 \%$ (21/206). Prevalence of ESBL production among the genders distribution was showed no significant difference $(\mathrm{p}=0.587)$ (Table 2).

\section{Multidrug resistance patterns of ESBL $+\mathrm{Ve} /-\mathrm{Ve}$ E. coli and K. pneumoniae}

All the ESBL producing isolates were 100\% resistant to all penicillin's, third-generation cephalosporins (e.g. Ceftazidime, Cefotaxime and Ceftriaxone) and Aztreonam. In ESBL positive E. coli $(\mathrm{n}=111)$, the most sensitive drug were Imipenem (99.0\%), Ertapenem (96.0\%), Nitrofurantoin $(92.0 \%)$, Cefoxitin $(86.0 \%)$ and resistant to Levofloxacin, Ciprofloxacin (96.0\% each), Tetracycline (78.0\%), Ampicillin/Sulbactum (77.0\%), Tobramycin, Cotrimoxazole $(76.0 \%$ each $)$ Figure 1. 
Table.1 Demographical features of the studies subjects

\begin{tabular}{|c|c|c|c|c|}
\hline Features & TOTAL (n) & Neg*(\%) & Pos** $(\%)$ & $p$-Value \\
\hline & 1495 & $1160(77.59$ & $335(22.41)$ & \multirow[t]{2}{*}{$\mathbf{N A}^{\#}$} \\
\hline MEDIAN AGE (RANGE) & $40(0-89)$ & $39(0-88)$ & $47(0-89)$ & \\
\hline \multicolumn{5}{|l|}{ AGE GROUP } \\
\hline $0-15$ & $196(13.11)$ & $156(79.59)$ & $40(20.41)$ & \multirow[t]{5}{*}{0.000} \\
\hline $16-30$ & $343(22.94)$ & $284(82.80)$ & $59(17.20)$ & \\
\hline $31-45$ & $320(21.40)$ & $255(79.69)$ & $65(20.31)$ & \\
\hline $46-60$ & $287(19.20)$ & $230(80.14)$ & $57(19.86)$ & \\
\hline$>60$ & $349(23.34)$ & $235(67.34)$ & $114(32.66)$ & \\
\hline \multicolumn{5}{|l|}{ GENDER } \\
\hline MALE & $637(42.61)$ & $530(83.20)$ & $107(16.80)$ & \multirow[t]{2}{*}{0.000} \\
\hline FEMALE & $858(57.39)$ & $630(73.43)$ & $228(26.57)$ & \\
\hline
\end{tabular}

*Neg- Negative, ${ }^{* * P o s-}$ Positive, ${ }^{\#}$ NA - Not applicable

Table.2 ESBL status of studied uropathogens

\begin{tabular}{|c|c|c|c|c|c|}
\hline Features & $\begin{array}{l}\text { Total } \\
\text { GNB }\end{array}$ & $\begin{array}{l}\text { ESBL+ve } \\
(\%)\end{array}$ & ESBL-ve (\%) & $\begin{array}{l}\text { Other } \\
\text { GNB* }(\%)\end{array}$ & p-Value \\
\hline & 303 & $118(38.94)$ & $145(47.85)$ & $40(13.20)$ & \multirow[t]{2}{*}{$\mathbf{N} \mathbf{A}^{\#}$} \\
\hline MEDIAN AGE (RANGE) & $49(0-89)$ & $54(0-89)$ & $45(0-86)$ & $47.5(1-88)$ & \\
\hline \multicolumn{6}{|l|}{ Organisms } \\
\hline E. coli & $234(77.23)$ & $111(47.44)$ & $123(52.56)$ & \multirow[t]{5}{*}{$\mathbf{N A ^ { \# }}$} & \multirow[t]{5}{*}{0.104} \\
\hline K. pneumoniae & $27(8.91)$ & $7(25.93)$ & $20(74.07)$ & & \\
\hline P. mirabilis & $1(0.33)$ & $0(0.0)$ & $1(100.0)$ & & \\
\hline K. oxytoca & $1(0.33)$ & $0(0.0)$ & $1(100.0)$ & & \\
\hline Others & $40(13.20)$ & $\mathbf{N A} \mathbf{A}^{\#}$ & $\mathbf{N A}^{\#}$ & & \\
\hline \multicolumn{6}{|l|}{ Gender } \\
\hline MALE & $97(32.01)$ & $37(38.14)$ & $41(42.27)$ & $19(19.59)$ & \multirow[t]{2}{*}{0.587} \\
\hline FEMALE & $206(67.99)$ & $81(39.32)$ & $104(50.49)$ & $21(10.19)$ & \\
\hline
\end{tabular}

*GNB- Gram Negative Bacteria, ${ }^{\#} \mathrm{NA}$ - Not applicable

Table.3 MDR Status of ESBL +/-ve E. coli and K. pneumoniae isolates

\begin{tabular}{|l|l|l|l|}
\hline Features & Total $(\boldsymbol{\%})$ & MDR* $(\%)$ & NON-MDR* (\%) \\
\hline Organisms & 261 & $206(78.93)$ & $55(21.07)$ \\
\hline E. coli & $234(89.66)$ & $189(80.77)$ & $45(19.23)$ \\
\hline K. pneumoniae & $27(10.34)$ & $17(62.96)$ & $10(37.04)$ \\
\hline ESBL POSITIVE & $\mathbf{1 1 8}(\mathbf{4 5 . 2 1})$ & $\mathbf{1 1 8}(\mathbf{1 0 0 . 0})$ & $\mathbf{0}(\mathbf{0 . 0 \%})$ \\
\hline E. coli & $111(94.07)$ & $111(100.0)$ & $0(0.0 \%)$ \\
\hline K. pneumoniae & $7(5.93)$ & $7(100.0)$ & $0(0.0 \%)$ \\
\hline ESBL NEGATIVE & $\mathbf{1 4 3 ( 5 4 . 7 9 )}$ & $\mathbf{8 8}(\mathbf{6 1 . 5 4})$ & $\mathbf{5 5}(\mathbf{3 8 . 4 6})$ \\
\hline E. coli & $123(86.01)$ & $78(63.41)$ & $45(36.59)$ \\
\hline K. pneumoniae & $20(13.99)$ & $10(50.0)$ & $10(50.0)$ \\
\hline
\end{tabular}

*MDR- Multi-Drug Resistant. 


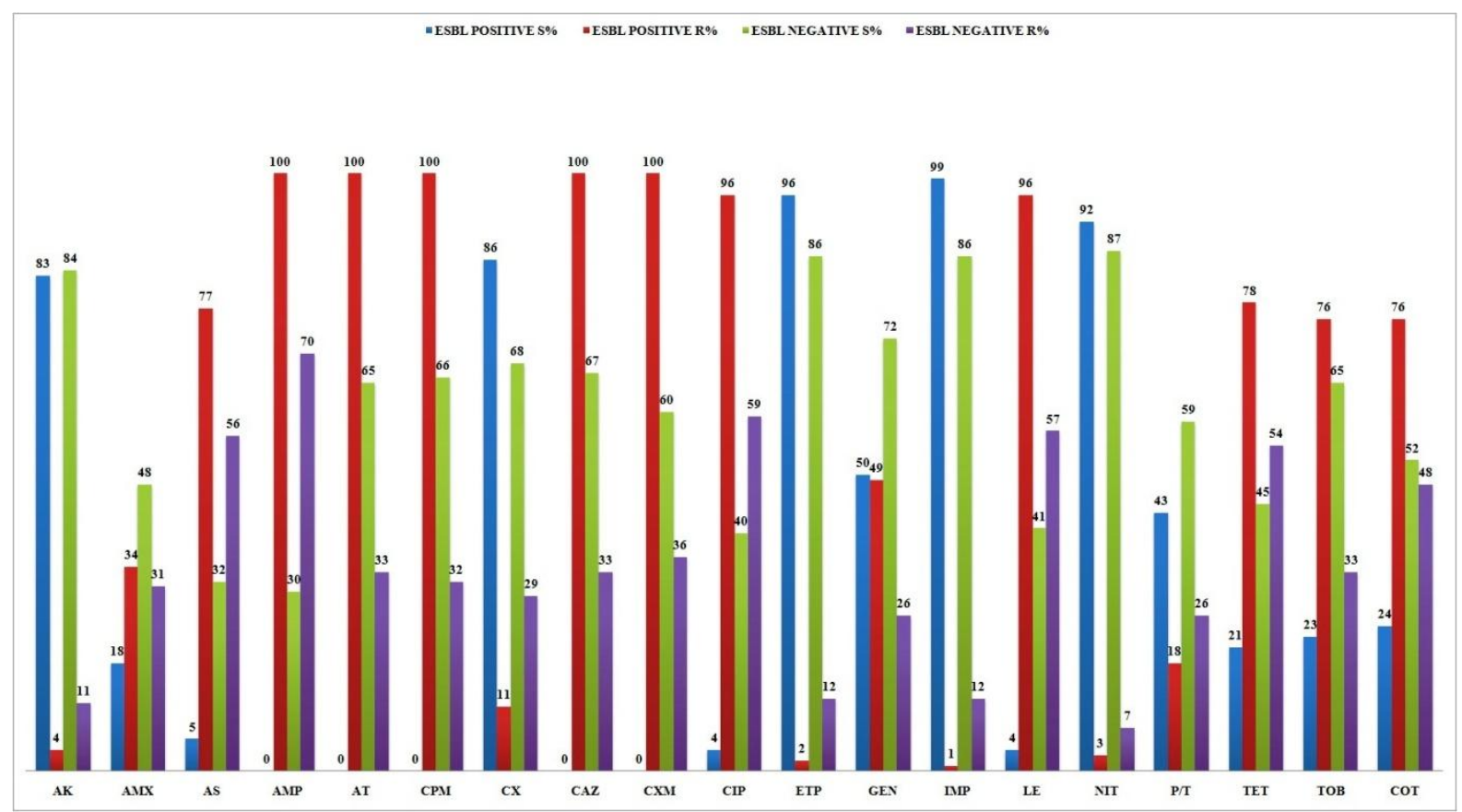

Fig.1 Antibiogram showing Sensitivity and Resistance Patterns of E. coli $S \%=$ Sesnsitivity; $R \%=$ Resistant

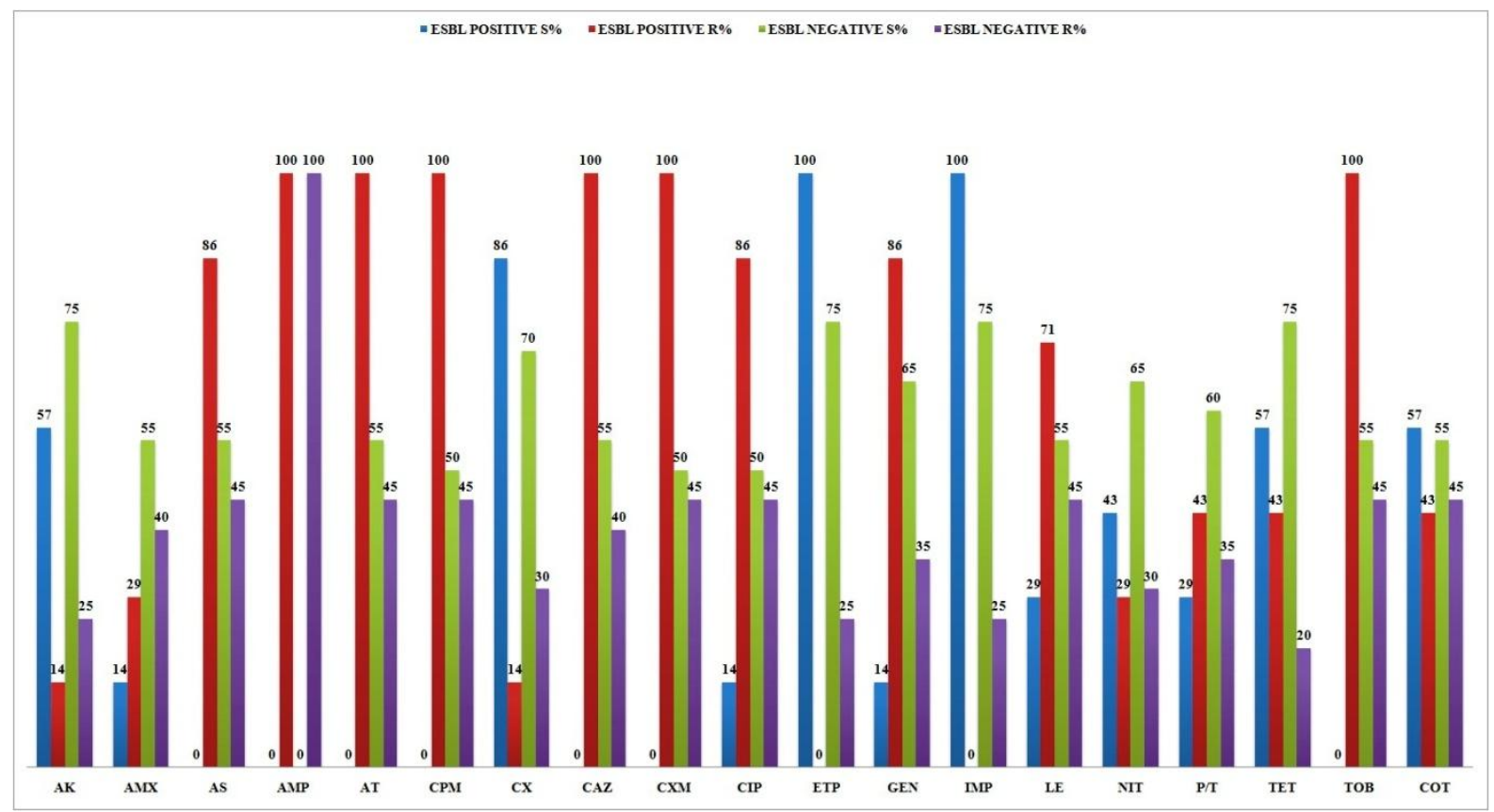

Fig.2 Antibiogram showing Sensitivity and Resistance Patterns of $K$. pneumoniae $S \%=$ Sensitivity; $R \%=$ Resistant

In ESBL positive $K$. pneumoniae $(\mathrm{n}=7)$, the most sensitive drugs were Imipenem, Ertapenem (100.0\% each), Cefoxitin (86.0\%), Tetracycline, Cotrimoxazole,
Amikacin (57.0\% each). The strain was resistant to Tobramycin (100.0\%), Ampicillin/Sulbactum, Ciprofloxacin, Gentamicin (86.0\% each), Levofloxacin 
(71.0\%), Piperacillin/ Tazobactum, Tetracycline, Cotrimoxazole (43.0\% each) (Figure 2). In ESBL negative E. coli $(\mathrm{n}=123)$, the most sensitive drug were Nitrofurantoin $(87.0 \%), \quad$ Ertapenem, Imipenem (86.0\% each), Amikacin (84.0\%), Gentamicin (72.0\%), and resistant to Ampicillin (70.0\%), Ciprofloxacin (59.0\%), Levofloxacin (57.0\%), Ampicillin/ Sulbactum (56.0\%) (Figure 1). In ESBL negative $K$. pneumoniae $(\mathrm{n}=20)$, the most sensitive drug were Amikacin, Ertapenem, Imipenem, Tetracycline $(75.0 \%$ each $)$, Cefoxitin (70.0\%), Nitrofurantoin, Gentamicin (65.0\% each), Piperacillin/ Tazobactam (60.0\%) followed by Amoxicillin/ clavulanic acid, Ampicillin/ Sulbactam, Aztreonam, Ceftazidime, Tobramycin, Levofloxacin, Cotrimoxazole (55.0\% each). The strain was resistant to Ampicillin (100.0\%), Ampicillin/Sulbactam, Cefuroxime, Aztreonam, Cefepime, Ciprofloxacin, Levofloxacin, Tobramycin, Cotrimoxazole (45.0\% each), Amoxicillin/ clavulanic acid, Ceftazidime ( $40.0 \%$ each), Gentamicin, Piperacillin/Tazobactam (35.0\% each) (Figure 2). The sensitivity and resistance patterns of other antibiotics are depicted in Figure 1 and 2.

In the current study, MDR were $78.93 \%$ (206/261), and Non-MDR were $21.07 \%$ (55/261). The frequency of $E$. coli isolates were $89.66 \%$ (234/261), and $K$. pneumoniae were $10.34 \%$ (27/261). Among the 261 isolates, $45.21 \%(118 / 261)$ were ESBL positive, and $54.79 \%$ (143/261) were ESBL negative. There were $80.77 \%(189 / 234)$ MDR E. coli and $19.23 \%$ (45/234) were non-MDR Escherichia coli. There were $62.96 \%$ (17/27) were MDR K. pneumoniae, and $37.04 \%(10 / 27)$ were non-MDR $K$. pneumoniae. In ESBL positive group 94.07\% (111/118) were E. coli and 5.93\% (7/118) were Klebsiella pneumoniae. ESBL positive E. coli and $K$. pneumoniae were found as multidrug-resistant (100\%). In ESBL negative group $67.33 \%$ (68/101) were E. coli and $32.67 \%$ (33/101) were Klebsiella pneumoniae. $63.41 \%$ (78/123) E. coli strains were MDR and $36.59 \%$ (45/123) were nonMDR. 50.0\% (10/20) Klebsiella strains were MDR, and 50.0\% (10/20) were non-MDR. (Table 3).

The study evaluated the resistant patterns of ESBL producing and non-producing uropathogens isolated from the nonhospitalized patients from Gurugram, Haryana. The study provides the valuable laboratory data and allows comparison of the situation in Haryana with other parts of the country.

The result showed that $22.41 \%$ of urine samples from the non-hospitalized patients yielded significant pathogens. In the current study, the positive culture rate was found to be higher than previous studies from Aligarh, India $(10.86 \%)^{4}$, Tehran, Iran $(6.3 \%)^{18}$. The last research done at Indore, Madhya Pradesh showed the higher prevalence $(30.0 \%)$ than the present study ${ }^{19}$. Females were found to be significantly prevalent to UTI as compared to males (Female: $26.57 \%$; Males: 16.80; $\mathrm{p}<0.005$ ). The findings were contradictory to the study done by Bajpai et al., which found male were more prevalent to UTI as compared to females (Males: $55.5 \%$; Females: 44.4\%) and in favour of studies done by Sasirekha et al., Manjunath et al., and Sood et al., ${ }^{20-22}$.

In the present study, E. coli $(44.74 \%)$ was found to be most frequent uropathogen with ESBL production followed by $K$. pneumoniae (25.93\%). Most of the studies have also revealed E. coli was the most frequently isolated uropathogen followed by

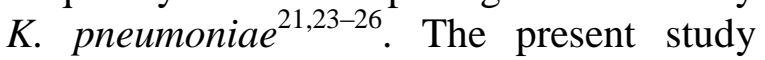
revealed 38.94\% ESBL producers which were in line with the survey conducted by 
previous research $(36.8 \%)^{19}$ and unlike the studies made by Khurana et al., (26.6\%), Tankhiwale et al., $(48.3 \%)^{27,28}$. The present study revealed $47.44 \%$ E. coli ESBL positive isolates resembling those reported by Bajpai et al., (41.66),Taneja et al., (40.02\%), Aruna and Mobashshera (49.32\%), Tankhiwale et al., (49.8\%) and Gururanjan et al., $(47.0 \%)^{19,24,28-30}$ and unlike those reported by Ramesh et al., (60.7\%), Singhal et al., (62.0\%), Kesavram et al., (59.1\%), Maya et al., $(75.5 \%)^{23,31-33}$. The reports presented by different authors indicated that the prevalence of ESBL production among clinical isolates vary significantly due to geographical differences and rapidly changing ESBL and sensitivity trends over time to time ${ }^{11,34}$. In the current study, ESBL production was seen almost similar in both the Genders (Males: 38.14 $\%$; Females: $39.32 \%$ ).

The present study reveals $25.93 \%$ of the $K$. pneumoniae isolates were ESBL producers which is very high with the study conducted by Sood et al., $(8.69 \%)^{22}$ whereas other studies had shown different ESBL production in Klebsiella pneumoniae. Aggarwal et al., reported $54.54 \%$ of Klebsiella species to be ESBL producers from Rohtak, Haryana ${ }^{35}$, in another study from Nagpur $25.6 \%{ }^{28}$ Klebsiella species were found to be ESBL producers which were in the line of our research. Pitout et al., has also highlighted the emergence of Enterobacteriaceae producing ESBLs in the community mainly from UTIs ${ }^{9}$. The geographical difference may be due to the use of different patterns of antibiotic usage. Our study confirms the Global trend towards the increased resistance to the $\beta$-lactam group of antibiotics. ESBL producing bacteria may not be detectable by routine disc diffusion sensitivity testing, leading to the inappropriate use of antibiotics and failure in the treatment. It is emphasized that Institutions should employ appropriate tests for their detection and avoid indiscriminate use of third-generation cephalosporins. The treatment should only be given after the culture and sensitivity test report to prevent the Multi-drug resistance.

Antibiotic resistance showed by different isolates is one of the barricades that might hinder a successful treatment. Widespread use of antibiotics exerts the selective pressure that acts as a driving force in the development of the resistance to various antibiotics ${ }^{19}$. The detailed insight of the antibiogram is illustrated in Figure 1 and 2 . Constant survey of the antibiotic-resistant pattern plays a very crucial role in the empiric treatment of UTIs.

A low degree of resistance to Amikacin $(E$. coli $\mathrm{ESBL}+\mathrm{ve}$ and $-\mathrm{ve}: 4 \%$ and $11 \% ; K$. pneumoniae ESBL +ve and -ve : $14 \%$ and $25 \%$ ) was observed for both ESBL and nonESBL producers and hence may be helpful in combating severe infections ${ }^{19,20,35}$. Aminoglycosides (Amikacin) being injectable is used restrictively in the community care settings and hence have shown lesser resistance rates ${ }^{22}$.

Resistance to antibiotics like Ampicillin (E. coli $\mathrm{ESBL}+\mathrm{ve}$ and -ve : $100 \%$ and $70.0 \%$; $K$. pneumoniae ESBL +ve and -ve : $100 \%$ each) and Cotrimoxazole (E. coli ESBL +ve and -ve : $76 \%$ and $48 \%$; K. pneumoniae ESBL +ve and -ve : $43 \%$ and $45 \%$ ) among ESBL producers and non-ESBL producers has been developed to such a level that prescribing them would lead to treatment failure. This can be predicted due to their overuse in the OPD patients without knowing their culture and sensitivity results $^{21}$.

An individual is at a significantly higher risk of being infected by the ESBL producing gram-negative organisms if he/she is exposed to antibiotics for a more extended period, suffers from a severe illness or an 
institute which is using third generations cephalosporin very frequently ${ }^{35}$. Under such circumferences, fluoroquinolones become drugs of choice. Levofloxacin and Ciprofloxacin showed $96 \%$ resistance to oral antibiotics in ESBL producers Escherichia coli. Levofloxacin had $71.0 \%$ and Ciprofloxacin had $86.0 \%$ resistance towards Klebsiella pneumoniae. This finding is consistent with the previous studies ${ }^{19,21,35}$. Since they are frequently prescribed in the OPD patients, it accounts for the emergence of resistance against them. Fluoroquinolones showed varied side effects when they are prescribed in the higher doses. Hence its extensive use must be avoided.

Another oral antibiotic Nitrofurantoin (ESBL positive E. coli: 3.0\%; ESBL positive $K$. pneumoniae: $29.0 \%$; ESBL negative E. coli:7.0\%; ESBL negative $K$. pneumoniae: $30 \%$ ) was found to be more effective in treatment of UTI in the present study area. The findings were in agreement with similar surveillance studies done by Bajpai et al., Sasirekha et al., and Khameneh et al. ${ }^{19,20,36}$ and other Indian studies, which have demonstrated nitrofurans as an appropriate agent for the first line treatment of the communityacquired $\mathrm{UTI}^{22,37,38}$. Low antimicrobial resistance for nitrofurans can be attributed to its localised action on urinary tract and not being exposed outside urinary tract ${ }^{39}$.

Resistant to Imipenem, which is used as last resort drug in the healthcare facilities were found to be $99 \%$ and $100 \%$ in both ESBL positive E. coli and $K$. pneumoniae. This may be because patients in community care are not directly being treated with Imipenem.

The resistance has been increased considerably, and it was primarily due to the excessive and unnecessary use of the antimicrobial agents for non-therapeutic complaints. The WHO guidelines recommend Cotrimoxazole and Ampicillin as the first choice of drugs for UTIs treatment. In contrast, these two antibiotics cannot serve as treatment of the choice in our regions due to high resistance in our study area.

Frequent unreasonable use of antibiotics changes the intestinal flora leading to the MDR in the pathogens ${ }^{40}$. In this study, we focused on the uropathogenic E. coli and $K$. pneumoniae strains and their antimicrobial sensitivity patterns to 10 different groups of antibiotics which were commonly administered to treat the UTIs. The organism resistant to three different antibiotic classes were considered as $\mathrm{MDR}^{41}$. Interestingly all Escherichia and $K$. pneumoniae ESBL positive isolates were found to be MDR. In the other studies, MDR was found in $92.5 \%$ among E. coli ${ }^{7}$. The level of MDR amongst the UTI isolates was found to vary from country to country; it was reported to be $7.1 \%$ in USA $^{42,43}$ while $42 \%$ of uropathogenic E. coli in Slovenia ${ }^{44}$.

In conclusion, the monitoring of antibiotic resistance and susceptibility of bacterial strains in the community should be mandatory due to the higher frequency of the ESBL producing uropathogens found in the non-hospitalized patients. It was proved in this study that the multi-drug resistance was responsible for the significant treatment failure. So testing of ESBL production in the uropathogens is warranted. It is also suggested to not prescribe any antibiotics without undertaking the culture and sensitivity report as it may further erode the antibiotics sensitivity in the studied region population.

The present study was first of its kind in our region. This has provided the holistic 
understanding of the ongoing trends of antibiotic sensitivity pattern of ESBL producing and non producing uropathogens. The highlight of the study was the identification of antibiotics which had failed in showing sensitivity in the in-vitro analysis. These antibiotics belonged to primarily the first line of antibiotics, which is progressively losing its sheen in local patient's health-care. Thus, this finding comes with information that such first-line antibiotics should not be used on patients of the region, and on it causes a big concern for us due to its failed sensitivity on the local population. This also implies that progressively these antibiotics are ineffective due to rampant use of them in Indian medicine system. It is worth mentioning that these observations are in line with national and international studies, who had been demanding strict prescription norms for the antibiotics.

\section{Acknowledgements}

The study was done at Bacteriology section, Department of Microbiology, Modern Diagnostic and Research Centre, Gurugram. We would like to express our special appreciation and thanks to all the patients whose samples were used in this study, my newborn angel Ivanka Rajput, wife and father-mother who gave me inspiration to write the research papers, technicians of my department, my beloved friends Dr. Ajay Soni, Dr. Siva Adarsh, Dr. Sangeet Bhaumik and our Director, Dr. D.S Yadav for his continuous guidance, constitutive criticism, and constant encouragement.

\section{References}

1. Schappert, S. M. and Rechtsteiner, E. A. National Health Statistics Reports, Number 8, (August 6, 2008). 6, (2006).

2. Tambekar, D. H., Dhanorkar, D. V,
Gulhane, S. R., Khandelwal, V. K. and Dudhane, M. N. Antibacterial susceptibility of some urinary tract pathogens to commonly used antibiotics. African J. Biotechnol. 5, 1562-1565 (2006).

3. Gonzalez, C. M. and Schaeffer, A. J. Treatment of urinary tract infection: what's old, what's new, and what works. World J. Urol. 17, 372-82 (1999).

4. Akram, M., Shahid, M. and Khan, A. U. Etiology and antibiotic resistance patterns of community-acquired urinary tract infections in $\mathrm{J} \mathrm{N} \mathrm{M} \mathrm{C} \mathrm{Hospital}$ Aligarh, India. Ann. Clin. Microbiol. Antimicrob. 6, 4 (2007).

5. Urine infection (UTI): symptoms and treatment. Available at: http://www.netdoctor.co.uk/conditions/li ver-kidney-and-urinarysystem/a12003/urinary-tract-infectionuti/. (Accessed: 30th December 2017)

6. Najar, M. S., Saldanha, C. L. and Banday, K. A. Approach to urinary tract infections. Indian J. Nephrol. 19, 12939 (2009).

7. Mukherjee, M., Basu, S., Mukherjee, S. K. and Majumder, M. MultidrugResistance and Extended Spectrum Beta-Lactamase Production in Uropathogenic E. coli which were Isolated from Hospitalized Patients in Kolkata, India. J. Clin. Diagn. Res. 7, 449-53 (2013).

8. Bradford, P. A. Extended-Spectrum Lactamases in the 21st Century: Characterization, Epidemiology, and Detection of This Important Resistance Threat. Clin. Microbiol. Rev. 14, 933951 (2001).

9. Pitout, J. D. D., Nordmann, P., Laupland, K. B. and Poirel, L. Emergence of Enterobacteriaceae producing extended-spectrum $\beta$ lactamases (ESBLs) in the community. 
J. Antimicrob. Chemother. 56, 52-59 (2005).

10. Soltani, R., Ehsanpoor, M., Khorvash, F. and Shokri, D. Antimicrobial susceptibility pattern of extendedspectrum $\quad \beta$-lactamase-producing bacteria causing nosocomial urinary tract infections in an Iranian referral teaching hospital. J. Res. Pharm. Pract. 3, 6-11 (2014).

11. Krishnakumar S, Rajan RA, Babu MM, B. V. Antimicrobial susceptibility pattern of Extended Spectrum of Beta Lactamase (ESBL) producing uropathogens from pregnant women. Indian J. Med. Healthc. 1, 2278-2966 (2012).

12. Bali, E. B., Açık, L. and Sultan, N. Phenotypic and molecular characterization of SHV, TEM, CTX-M and extended-spectrum -lactamase produced by Escherichia coli, Acinobacter baumannii and Klebsiella isolates in a Turkish hospital. African $J$. Microbiol. Res. 4, 650-654 (2010).

13. Kiratisin, P., Apisarnthanarak, A., Laesripa, C. and Saifon, P. Molecular Characterization and Epidemiology of Extended-Spectrum- -LactamaseProducing Escherichia coli and Klebsiella pneumoniae Isolates Causing Health Care-Associated Infection in Thailand, Where the CTX-M Family Is Endemic. Antimicrob. Agents Chemother. 52, 2818-2824 (2008).

14. Dhillon, R. H.-P. and Clark, J. ESBLs: A Clear and Present Danger? Crit. Care Res. Pract. 2012, 625170 (2012).

15. Livermore, D. M. beta-lactamases in laboratory and clinical resistance. Clinical Microbiology Reviews 8, 557584 (1995).

16. Gholipour, A. et al., Phenotypic and Molecular Characterization of Extended-Spectrum $\quad \beta$-Lactamase Produced by Escherichia coli, and
Klebsiella pneumoniae Isolates in an Educational Hospital. Jundishapur J. Microbiol. 7, (2014).

17. CLSI. Performance Standards for Antimicrobial Susceptibility Testing; Nineteenth Informational Supplement. CLSI document M100-S19. Wayne, PA. USA. 29, (2009).

18. Kashef, N., Djavid, G. E. and Shahbazi, S. Antimicrobial susceptibility patterns of community-acquired uropathogens in Tehran, Iran. J. Infect. Dev. Ctries. 4, 202-6 (2010).

19. Bajpai, T., Pandey, M., Varma, M. and Bhatambare, G. S. Prevalence of extended spectrum beta-lactamase producing uropathogens and their antibiotic resistance profile in patients visiting a tertiary care hospital in central India: Implications on empiric therapy. Indian J. Pathol. Microbiol. 57, 407-12 (2014).

20. Sasirekha, B. Prevalence of ESBL, AmpC $\beta$-lactamases and MRSA among uropathogens and its antibiogram. EXCLI J. 12, 81-8 (2013).

21. Manjunath GN, Prakash R, Vamseedhar Annam, K. S. Changing trends in the spectrum of antimicrobial drug resistance pattern of uropathogens isolated from hospitals and community patients with urinary tract infections in Tumkur and. Int. J. Biol. Med. Res. 2, 504-507 (2011).

22. Sood, S. and Gupta, R. Antibiotic resistance pattern of community acquired uropathogens at a tertiary care hospital in jaipur, rajasthan. Indian $J$. Community Med. 37, 39-44 (2012).

23. Ramesh N, Sumathi CS, Balasubramanian V, Palaniappan KR, K. V. Urinary Tract Infection and Antimicrobial Susceptibility Pattern of Extended Spectrum of Beta Lactamase Producing Clinical Isolates. Adv. Biol ogical Res. 2, 78-82 (2008). 
24. Aruna, K. and Mobashshera, T. Prevalence of extended spectrum betalactamase production among uropathogens in south Mumbai and its antibiogram pattern. EXCLI J. 11, 36372 (2012).

25. Gales, A. C., Sader, H. S., Jones, R. N. and SENTRY Participants Group (Latin America). Urinary tract infection trends in Latin American hospitals: report from the SENTRY antimicrobial surveillance program (1997-2000). Diagn. Microbiol. Infect. Dis. 44, 289-99 (2002).

26. Mathur, P., Kapil, A., Das, B. and Dhawan, B. Prevalence of extended spectrum beta lactamase producing gram negative bacteria in a tertiary care hospital. Indian J. Med. Res. 115, 153-7 (2002).

27. Khurana, S., Taneja, N. and Sharma, M. Extended spectrum beta-lactamase mediated resistance in urinary tract isolates of family Enterobacteriaceae. Indian J. Med. Res. 116, 145-9 (2002).

28. Tankhiwale, S. S., Jalgaonkar, S. V, Ahamad, S. and Hassani, U. Evaluation of extended spectrum beta lactamase in urinary isolates. Indian J. Med. Res. 120, 553-6 (2004).

29. Taneja, N., Rao, P., Arora, J. and Dogra, A. Occurrence of ESBL andamp; AmpC beta-lactamases andamp; susceptibility to newer antimicrobial agents in complicated UTI. Indian $J$. Med. Res. 127, 85-8 (2008).

30. Gururajan, G., A. Kaliyap, K. and Ramasamy, B. Prevalence of Extended Spectrum Beta Lactamases in Uropathogenic Escherichia coli and Klebsiella Species in a Chennai Suburban Tertiary Care Hospital and its Antibiogram Pattern. Res. J. Microbiol. 6, 796-804 (2011).

31. Singhal, S. et al., Evaluation of methods for AmpC beta-lactamase in gram negative clinical isolates from tertiary care hospitals. Indian J. Med. Microbiol. 23, 120-4 (2005).

32. Kesavaram, P. et al., Detection of Extended-Spectrum $\beta$ Lactamases and AmpC $\beta$-Lactamase Production in Escherichia coli Causing Urinary Tract Infection among HIV and Non-HIV Patients. Am. Med. J. 2, 54-58 (2011).

33. Maya AS, Prabhakar K, Hanna LE, S. Y. Phenotypic and Genotypic Characterization of Extended Spectrum b-Lactamase Producing Escherichia coli Clinical Isolates from Semiurban Area. J. Pharm. Res. 44, 6-10 (2011).

34. Babypadmini, S. and Appalaraju, B. Extended spectrum -lactamases in urinary isolates of Escherichia coli and Klebsiella pneumoniae - prevalence and susceptibility pattern in a tertiary care hospital. Indian J. Med. Microbiol. 22, 172-4 (2004).

35. Chaudhary, U. and Aggarwal, R. Extended spectrum -lactamases (ESBL) - an emerging threat to clinical therapeutics. Indian J. Med. Microbiol. 22, 75-80 (2004).

36. Khameneh, Z. R. and Afshar, A. T. Antimicrobial susceptibility pattern of urinary tract pathogens. Saudi J. kidney Dis. Transplant. 20, 251-3 (2009).

37. Kothari, A., Sagar, V. and Sagar, V. Antibiotic resistance in pathogens causing community-acquired urinary tract infections in India: a multicenter study. J. Infect. Dev. Ctries. 2, 354-358 (2008).

38. Biswas, D. et al., Choice of antibiotic for empirical therapy of acute cystitis in a setting of high antimicrobial resistance. Indian J. Med. Sci. 60, 53-8 (2006).

39. Rajesh KR, Mathavi S, I. R. Prevalence Of Antimicrobial Resistance In Uropathogens And Determining Empirical Therapy For Urinary Tract 
Infections. Int. J. Basic Med. Sci. 1, 260-263 (2010).

40. Soto, S. M., Jimenez de Anta, M. T. and Vila, J. Quinolones Induce Partial or Total Loss of Pathogenicity Islands in Uropathogenic Escherichia coli by SOS-Dependent or -Independent Pathways, Respectively. Antimicrob. Agents Chemother. 50, 649-653 (2006).

41. Wilson, A. P. R. et al., Prevention and control of multi-drug-resistant Gramnegative bacteria: recommendations from a Joint Working Party. J. Hosp. Infect. 92, S1-S44 (2016).

42. Gonzales, R., Malone, D. C., Maselli, J. H. and Sande, M. A. Excessive
Antibiotic Use for Acute Respiratory Infections in the United States. Clin. Infect. Dis. 33, 757-762 (2001).

43. Linder, J. A., Huang, E. S., Steinman, M. A., Gonzales, R. and Stafford, R. S. Fluoroquinolone prescribing in the United States: 1995 to 2002. Am. J. Med. 118, 259-268 (2005).

44. Rijavec, M. et al., High Prevalence of Multidrug Resistance and Random Distribution of Mobile Genetic Elements Among Uropathogenic Escherichia coli (UPEC) of the Four Major Phylogenetic Groups. Curr. Microbiol. 53, 158-162 (2006).

\section{How to cite this article:}

Rishabh Rajput and Surendra Sarsaiya. 2018. Significance of Regional Antibiogram and MDR of ESBL Producing Uropathogens Infecting Non-hospitalized Patients: Gurugram. Int.J.Curr.Microbiol.App.Sci. 7(02): 1114-1126. doi: https://doi.org/10.20546/ijcmas.2018.702.139 